
allemande

47-1| 2015

L'Allemagne et l'Europe | La déprise de l'Empire napoléonien en Allemagne en 1813

\title{
Mémoires, mythes et relectures de la bataille de Leipzig en Allemagne de 1813 à 1871
}

Jérôme Schweitzer

\section{(2) OpenEdition}

\section{Journals}

Édition électronique

URL : https://journals.openedition.org/allemagne/477

DOI : 10.4000 /allemagne.477

ISSN : 2605-7913

Éditeur

Société d'études allemandes

Édition imprimée

Date de publication : 26 juin 2015

Pagination : 185-194

ISSN : 0035-0974

Référence électronique

Jérôme Schweitzer, « Mémoires, mythes et relectures de la bataille de Leipzig en Allemagne de 1813 à 1871 », Revue d'Allemagne et des pays de langue allemande [En ligne], 47-1 | 2015, mis en ligne le 13 décembre 2017, consulté le 19 mai 2021. URL : http://journals.openedition.org/allemagne/477 ; DOI : https://doi.org/10.4000/allemagne.477 


\title{
Mémoires, mythes et relectures de la bataille de Leipzig en Allemagne de 1813 à 1871
}

\author{
- Jérôme Schweitzer*
}

En octobre 1813, la nouvelle de la défaite subie par Napoléon à Leipzig a un retentissement sans précédent en Allemagne. Suivant le modèle instauré par Napoléon, les armées de la Coalition font publier des bulletins afin d'informer les populations des avancées de leurs troupes. Ces textes sont largement diffusés dans le sillage des soldats qui s'élancent vers le Rhin ou paraissent dans les multiples journaux apparus en Allemagne dans une période de relative liberté de la presse ${ }^{(1)}$. Dans ce contexte, ces publications contribuent à élaborer la première vision de ces combats rapidement appelés les guerres de libération ${ }^{(2)}$. Même s'il faut nuancer la portée de ces textes, des études récentes ayant souligné que le lectorat sensible aux idées nationalistes était relativement limité(3) une forme indéniable de fascination se développe pour le récit de la bataille de Leipzig dès la fin du mois d'octobre 1813. Ainsi, le Leipziger Tageblatt du $1^{\mathrm{er}}$ novembre 1813 souligne:

«Ces jours, si importants pour l'Europe, resplendiront dans les annales du monde, les dixhuit et dix-neuf octobre resteront inoubliables pour l'Allemagne. La génération future sera

* Archiviste paléographe, conservateur à la Bibliothèque nationale et universitaire de Strasbourg.

1 Kurt Koszy K, Geschichte der deutschen Presse, vol. 2, Berlin, Colloquium, 1966, p. 25-27.

2 Voir à ce propos: Kirstin Anne Schäfer, «Völkerschlacht», in: Étienne François, Hagen Schulze (dir.), Deutsche Erinnerungsorte, t. 2, Munich, C. H. Beck, 2001, p. 187-201; Helmut Berding, «Das Geschichtliche Problem der Freiheitskriege (1813-1814) ", in: Karl Otmar von Aretin, Gerhard RitTER (dir.), Historismus und moderne Geschichtswissenschaft, Europa zwischen Revolution und Restauration (1797-1815), Drittes Deutsch-Sowjetisches Historikertreffen in der Bundesrepublik Deutschland München, 13-18 März 1978, Stuttgart, Steiner, 1987, p. 201-215.

3 Ute Planert, Der Mythos vom Befreiungskrieg, Frankreichs Kriege und der deutsche Süden: AlltagWahrnehmung-Deutung (1792-1841), Paderborn, Schöningh, 2007, p. 477: l'auteur rappelle que la majorité de la population rurale était analphabète, que la plupart des bourgeois ou nobles sensibles aux idées nationalistes habitaient les villes et que par conséquent la diffusion de ce type d'idées fut plus complexe que l'image qui en a été faite au $\mathrm{XIX}^{\mathrm{e}}$ siècle. 
émerveillée lorsqu'elle visitera la ville où les forces combinées des plus nobles princes d'Europe accomplirent ce que nous tenions pour impossible par manque de courage, [...] ${ }^{(4)}$.

\section{Premiers échos, premières récupérations idéologiques}

Dans la majorité des récits de la bataille de Leipzig, la lecture politique des événements prend progressivement le pas sur la description des manœuvres militaires. Un autre journal, l'Europäische Aufseher, s'empare de ce thème dès la fin octobre 1813. Tout au long de l'année 1814, divers articles traitent de la bataille de Leipzig et de ses retombées. Le rédacteur en chef, $\operatorname{Bergk}^{(5)}$, en fait le symbole de la libération de l'oppression:

« Guerre aux Français! Voilà, le mot d'ordre qui s'est répandu dans toute l’Allemagne, [...]. Le combat doit continuer jusqu'à la libération de l'Europe et jusqu'à ce qu'elle soit protégée contre toute tentative sournoise de conquête, jusqu'à ce que l'Allemagne recouvre ses frontières dans lesquelles l'esprit humain s'exprime en langue germanique, jusqu'à ce que le genre humain soit protégé contre toute menace de monarchie universelle et jusqu'à ce que les peuples européens aient retrouvé la paix et la sécurité par le retour de l'équilibre politique ${ }^{(6)}$.

Passé ces réactions immédiates, une réflexion s'engage sur la meilleure manière d'entretenir le souvenir de ces journées d’octobre 1813. Pierre Nora a souligné « la nécessité d'organiser des célébrations, de prononcer des éloges funèbres, de notarier des actes, parce que ces opérations ne sont pas naturelles " ${ }^{(7)}$, car il n'y a pas de " mémoire spontanée ». Ce phénomène est à l'œuvre à Leipzig devenu l'objet de lectures à la fois matérielles et symboliques. Le théâtre de la «bataille des nations » acquiert une " aura symbolique » forte: pour les auteurs nationalistes il devint le lieu de naissance de la nation allemande. Dès le 30 octobre 1813, une cérémonie d'hommage aux combattants victorieux est organisée à Leipzig ${ }^{(8)}$. Affirmant cette nécessaire commémoration, le publiciste nationaliste Ernst Moritz Arndt s'exprime dans une brochure, Ein Wort über die Feier der Leipziger Schlacht, en septembre 1814. Dans cette publication, il décrit la manière dont la fête devrait être organisée à travers l'Allemagne afin de symboliser au mieux l'unité allemande. Il invite donc les Allemands à allumer de grands bûchers à travers l'ensemble du pays:

«Dès qu'il fera sombre, dans les prés de toute l'Allemagne, de Stralsund à Trèves et de Memel à Luxembourg, des feux s'allumeront au sommet des montagnes et là où il n'y en a pas, sur les collines, les hauteurs ou les tours, et ils seront alimentés jusqu'aux environs de minuit. Ils seront des messagers dans le lointain, un signe d'amour et de joie, annonçant

4 «Empfindungen bey dem allgemeinen Sieges- und Dankfeste zu Leipzig, den 31. Oktober 1813 », Leipziger Tageblatt, 305 (1813), p. 1217-1220, ici p. 1220.

5 Johann Heinrich Bergk (1769-1834) est un publiciste qui vécut la majorité de sa vie à Leipzig. Il utilisait parfois le pseudonyme Hainichen. Il a été rédacteur en chef des journaux l'Europäische Aufseher (fondé en 1805) et l'Europäische Beobachter (1808-1809), deux publications anti-françaises.

6 «Aehrenlese auf den Schlachtfeldern bei Leipzig », Europäische Aufseher, 1 (1814), p. 13-16, ici p. 15.

7 Pierre Nora (dir.), Les lieux de mémoire, t. 1, Paris, Gallimard, 1987, p. XXIV.

8 «Feyer des Dankfestes am 30sten Oktober, nach dem von den hohen allierten Mächten am 18sten und 19sten Oktober höchstglorreichen erfochtenen Siege bey Leipzig ", Leipziger Tageblatt, 308 (1813), p. 1229-1232, ici p. 1229. 
à tous ceux qui nous entourent qu'il ne règne désormais chez tous les Allemands qu'un sentiment et qu'une seule pensée " ${ }^{(9)}$.

L'appel lancé par Arndt s'est traduit dans les faits en octobre 1814. Le recueil, Des Teutschen Volkes feuriger Dank- und Ehrentempel ${ }^{(10)}$, publié en 1815, a recensé quasiment toutes les fêtes organisées à cette occasion. L'unité n'est cependant pas parfaite et Arndt dénonce « ceux qui avaient décidé de demeurer dans les ténèbres ». Même si elles n’ont pas eu lieu partout, ces célébrations sont révélatrices de la conception de l'émergence de la nation allemande développée par le courant nationaliste en opposition à la nation française ${ }^{(11)}$. La fête des 18 et 19 octobre 1814 est considérée comme l'une des premières fêtes nationales allemandes ${ }^{(12)}$. Malgré le caractère populaire mis en avant dans les journaux de l'époque, elle demeure une manifestation voulue par une petite élite essentiellement bourgeoise favorable aux idées nationalistes ${ }^{(13)}$. Parfois, la fête populaire a même été récupérée au profit d'une lecture favorable au pouvoir des monarques mis en difficulté par les idéaux de la Révolution française ${ }^{(14)}$.

Pour donner un caractère encore plus durable au souvenir de Leipzig, des projets de monuments commémoratifs voient le jour sous la plume de l'écrivain Adolph von Seckendorff ou d'Arndt lui-même ${ }^{(15)}$. Autre signe de l'importance accordée aux événements: dès 1814 , des habitants de Leipzig créent l'Association du 19 octobre ${ }^{(16)}$. Leur ambition consiste à encourager et faire perdurer le souvenir de la bataille en soutenant la construction de monuments ou l'organisation d'une commémoration annuelle. L'une des premières actions de l'association se traduit par l'édification d'une imposante croix en bois à Probstheyda ${ }^{(17)}$, le lieu où les combats furent les plus durs lors du 18 octobre 1813. Abattue par des inconnus, cette croix mit en lumière les tensions liées

9 Ernst Moritz Arndt, Ein Wort über die Feier der Leipziger Schlacht, Francfort, P. W. Eichenberg, 1814, p. 11.

10 Karl Hoffmann, Des Teutschen Volkes feuriger Dank-und Ehrentempel oder Beschreibung wie das aus zwanzigjähriger französischer Sklaverei durch Fürsten-Eintracht und Volkskraft gerettete Teutschen Volk die Tage der entscheidenden Völker-und Rettungsschlacht bei Leipzig am 18. und 19. Oktober 1814 zum erstenmale gefeiert hat, Offenbach, Brede, 1815, X-11, 46 p.

11 Dieter Düding, « Das deutsche Nationalfest von 1814; Matrix der deutschen Nationalfeste im 19. Jahrhundert », in: D. Düding, Peter Friedemann, Paul Munch (dir.), Öffentliche Festkultur, politische Feste in Deutschland von der Aufklärung bis zum Ersten Weltkrieg, Hambourg, Rowohlt, 1988, p. 67-84.

12 Ibid., p. 84.

13 Ibid., p. 84: la fête devait « ouvrir la voie à un État allemand qui serait né avec l'aide des princes et non contre eux »; Uwe Puschner, « Der 18. Oktober, zur Karriere eines Tages im 19. Jahrhundert ", in: Pierre BÉHAR, Michel Grunewald (dir.), Frontières, transferts, échanges transfrontaliers et interculturels: Actes du XXXVI congrès de l'Association des Germanistes de l'Enseignement Supérieur, Berne, Lang, 2005, p. 139-155.

14 U. Planert, Der Mythos vom Befreiungskrieg (note 3), p. 618.

15 E. M. Arndt, «Über ein Denkmal bei Leipzig », in: E. M. Arndt, Ein Wort über die Feier (note 9), p. 20-22.

16 «Erste Jahresfeyer des 19sten Oktobers 1813, als des denkwürdigen Tages der Errettung Leipzig, von den Bürgern und Einwohnern Leipzigs zu Probstheyda am 19. Okt. 1814 begangen ", Leipziger Tageblatt, 305 (1814), p. 1217-1220.

17 Ibid. 
au souvenir de la bataille de Leipzig en Saxe. Un placard portant la mention « Les Prussiens nous ont volé notre territoire, le diable peut bien nous voler une croix » aurait été retrouvé sur les lieux par la suite ${ }^{(18)}$. Quelques années plus tard, en 1818, un article paru dans le journal saxon Allgemeinen Anzeiger der Deutschen est revenu sur ces événements en soulignant que certains Saxons abhorraient cette croix symbole de "leur triste sort » ${ }^{(19)}$. Loin d'y voir le signe de la libération du « joug français », les Saxons ne retenaient que l'abaissement de leur pays. En effet, la Saxe a tenu une place ambiguë durant la bataille de Leipzig puisque son roi est resté fidèle à Napoléon jusqu'à la fin, alors que ses troupes ont subitement changé de camp le troisième jour des combats.

De fait, cette situation particulière place la Saxe en porte-à-faux vis-à-vis des autres États allemands, dans la mesure où officiellement le royaume est demeuré du côté français (raison pour laquelle il fut amputé de nombreux territoires au profit de la Prusse lors du congrès de Vienne de 1815). Ce souvenir douloureux pour la Saxe marque une première rupture dans l'unité allemande appelée de leurs vœux par les nationalistes. La réaction conservatrice de la fin des années 1810 met à bas les derniers espoirs des partisans de l'union des Allemands. Le 18 octobre 1817, lors du quatrième anniversaire de la bataille et des trois cents ans des 95 thèses de Martin Luther, la fête de la Wartburg est la dernière grande fête qui commémore Leipzig. Les étudiants d’Iéna sont contraints d'admettre que " les grandes espérances " qui s'étaient élevées quatre ans plus tôt ne s'étaient pas réalisées ${ }^{(20)}$. Après cet événement, Metternich dénonce «les charlataneries de germanité, de liberté, de constitution, d’unité, etc. » ${ }^{(21)}$. Dès lors, les nationalistes sont pourchassés et mis à l'écart. La presse est davantage surveillée: de fait l'interprétation de la bataille de Leipzig est désormais bien encadrée.

\section{De 1818 aux années 1860, un souvenir entre oubli et récupération monarchique}

Après 1815, la restauration du pouvoir monarchique s'impose en Europe. Dans ce contexte, le souvenir de la bataille de Leipzig n'échappe pas à une réinterprétation dans un sens plus conforme aux ambitions politiques des différents princes allemands ${ }^{(22)}$. Ute Planert a souligné ces points de vue divers développés entre 1815 et les années $1840^{(23)}$ : l'unité politique allemande paraît alors bien lointaine. De surcroît, la lecture de ces conflits n'est pas univoque en Allemagne au XIX ${ }^{\mathrm{e}}$ siècle: elle est fortement dépendante des divers intérêts politiques en jeu.

Après 1818, la bataille de Leipzig conserve un statut à part des autres combats de la période napoléonienne en Allemagne. Le discours mettant en avant l'union des Allemands contre un ennemi commun est abandonné au profit de la défense des

18 Stefan Poser, Denkmale zur Völkerschlacht, Leipzig, Passage, 2007, 160 p.

19 Ernst Teutmund, "Auch ein Wort über die Zerstörung des Kreuzes bey Probsthaida von einem gebornen Leipziger, der weder an der Errichtung, noch dem Umstirze Theil hatte ", Allgemeinen Anzeiger der Deutschen, 282 (1818), p. 3045-3052, ici p. 3048.

20 É. François, "La Wartburg », in: Étienne François, Hagen Schulze (dir.), Mémoires allemandes, Paris, Gallimard, 2007, p. 145-166.

21 Ibid.

22 H. Berding, "Das Geschichtliche Problem » (note 2), p. 202.

23 U. Planert, Der Mythos vom Befreiungskrieg (note 3), p. 620-640. 
intérêts des principautés allemandes. La Bavière et la Prusse se distinguent dans cette reprise en main mémorielle avec l'ambition d'unir leurs peuples derrière leur prince. Maximilien $\mathrm{I}^{\mathrm{er}}$, roi de Bavière, décide de suivre l'exemple de la Révolution française en rendant un hommage national aux soldats morts au combat. Dès 1817, il demande à son architecte, Léo von Klenze, de dessiner les plans d'un monument en souvenir des soldats bavarois tombés lors de la bataille de Polotsk en $1812^{(24)}$. Son fils, Louis I $^{\text {er }}$, suit cet exemple, en 1833. Lors du vingtième anniversaire de la bataille de Leipzig, il fait édifier un obélisque à Munich ${ }^{(25)}$. Les noms des 30000 soldats bavarois morts entre le début de la campagne de Russie et Waterloo sont gravés sur le monument. Le fait qu'avant octobre 1813 ces hommes soient morts pour Napoléon est passé sous silence, il importe alors d'incarner l'unité de la nation bavaroise derrière son roi. Les publications accompagnant l'inauguration soulignent que dès la campagne de Russie, la Bavière combattait " pour la patrie et la liberté des Allemands " ${ }^{(26)}$.

En Prusse, l'un des premiers États à changer d'alliance en 1812, Leipzig est considérée comme une bataille parmi d'autres ${ }^{(27)}$. Craignant de favoriser le nationalisme allemand et le libéralisme, le gouvernement préfère mettre en avant l'ensemble des guerres menées contre Napoléon. L’un des exemples de cette volonté politique est le monument inauguré à Berlin le 30 mars 1821 (date anniversaire de la bataille de Montmartre). Euvre de Schinkel qui s'inspira de l'architecture gothique, il est constitué de statues incarnant les batailles des années 1813-1815 réunies autour d'une flèche de cathédrale. L'édifice confère la même importance à chacun des combats.

Cette politique d'encadrement de la mémoire de Leipzig a un impact sur le champ de bataille d'octobre 1813 lui-même: les commémorations annuelles se font plus discrètes et n'obtiennent qu'une audience locale. Autre conséquence: l'Association du 19 octobre périclite avant d'être dissoute en $1827^{(28)}$. En 1820, lors de la cérémonie, un orateur regrette cette situation:

« L'Allemagne ne fut digne de la bataille de Teutobourg que durant le temps où elle la commémorait, ensuite elle l'a oubliée, oubliant en même temps que c'est cette lutte qui lui avait permis de défendre son indépendance face à l'empire romain ${ }^{(29)}$.

L'auteur de cette harangue dénonce également la désunion des Allemands, mais il ne peut que constater son impuissance face aux intérêts divergents des monarques. L'un des exemples le plus marquant de cette atonie dans laquelle sombre le souvenir de la bataille de Leipzig est sans doute la commémoration de son vingt-cinquième anniversaire en 1838. La fête est très modeste, dans les discours la liberté reconquise en 1813

24 Ibid., p. 627-628.

25 Ibid.

26 Ibid.

27 Hans-Ernst Mittig, "Die Sprache der Denkmäler », in: Düding/ Friedemann/ Munch (dir.), Öffentliche Festkultur (note 11), p. 20-41.

28 Robert Naumann, Die Völkerschlacht bei Leipzig, nebst Nachrichten von Zeitgenossen und Augenzeugen über dieselbe, im auftrage von dem Verein zur feier des 19. Oktober in Leipzig, Leipzig, Weigel, 1863, p. V.

29 Karl von Hase, Am Jahrestage von Leipzig: Rede, sieben Jahre nach der Völkerschlacht, Leipzig, Breitkopf \& Härtel, 1913, p. 8. 
est mise en avant, mais également le sort de la Saxe amputée au profit de la Prusse ${ }^{(30)}$. L'enthousiasme de 1813 semble alors avoir totalement disparu et la bataille de Leipzig est en passe de sombrer dans l'oubli. L'essor de la légende napoléonienne en Europe a contribué à enrayer cette destinée qui, en 1838, paraît inéluctable.

Après la mort de Napoléon en 1821, puis le retour des cendres en 1840, la fascination pour l'épisode napoléonien reprend le dessus. Alors qu'en France, Louis-Philippe décide d'achever les travaux de l'Arc-de-Triomphe en le dédiant aux victoires de la Révolution et de l'Empire, en Allemagne un regain d'intérêt pour la période de l'Empire se fait aussi sentir. Dans un premier temps, l'héroïsme des combats et les libertés nées de la Révolution sont mis en avant. Par la suite, les bouleversements politiques des années 1840 se servent de cette légende naissante afin d'asseoir la légitimité des aspirations nouvelles en développement.

L'Association du 19 octobre est ainsi rétablie en 1843 à Leipzig ${ }^{(31)}$. Son objectif demeure la commémoration annuelle de la bataille et la lutte contre l'oubli. Cela se traduit notamment par le recueil de témoignages et de souvenirs des derniers témoins des événements. Autre action mise en œuvre par l'association : l'édification de monuments commémoratifs sur les points les plus marquants du champ de bataille. Le 19 octobre 1847, une stèle est placée sur le Monarchenhügel, lieu où le roi de Prusse, l'empereur d'Autriche et le tsar apprirent la nouvelle de leur victoire sur Napoléon. En 1858, l'association ajoute deux nouvelles plaques, l'une à Möckern, l'autre sur le Galgenberg près de Wachau là où se trouvait Napoléon le 16 octobre. En 1857, un monument nommé le Napoleonstein fut inauguré sur la colline dite du Thonberg ${ }^{(32)}$ : site où le quartier général du chef de guerre français s'était installé en 1813. Dans le contexte immédiat du Printemps des peuples de 1848, le souvenir de la bataille met donc en avant les sites importants des combats mais aussi le souvenir de l'empereur des Français ${ }^{(33)}$. Il s'agit d'une évolution du souvenir de Leipzig, le rejet de ce qui rappelle la France ou son empereur est remplacé par la fascination pour Napoléon ou les points les plus marquants de la confrontation.

Au début des années 1860, ces évolutions entraînent la redécouverte du champ de la bataille en tant que lieu de mémoire. Le maire de Leipzig, Theodor Appel, décide de faire ériger des bornes sur l'ensemble du champ de bataille entre 1861 et 1864. Leur fonction consiste à figurer précisément les lieux où se trouvaient les corps d'armée des troupes de la Coalition et de la Grande Armée. Un guide de visite destiné aux voyageurs permettait de savoir précisément ce qui s'était passé là où ils se trouvaient. Dans cet opuscule, Appel, inspiré des récits homériques ou de l'Antiquité écrit:

"Déjà les héros de cette époque ont acquis l'éclat des récits mythiques et des histoires héroïques. Quelle joie n'aurait pas tout amateur d'Histoire s'il pouvait déchiffrer une stèle

30 Worte eines Sachsen zur fünf und zwanzigjährigen Gedächtnisfeier, der Schlacht bei Leipzig, Dresde, Arnold, 1838, p. 5.

31 R. Naumann, Die Völkerschlacht bei Leipzig, nebst Nachrichten (note 28), p. VI.

32 Ibid.

33 Olivier Hemmerle, « De l'Allemagne divisée: les monuments pour ou contre l'empereur Napoléon ", in: Marcel Watelet, Pierre Couvreur, Philippe de Villelongue (dir.), Waterloo, monuments et représentations de mémoires européennes (1792-2001), Louvain-la-Neuve, Association franco-européenne de Waterloo, 2003, p. 127-139. 
indiquant l'emplacement de la tente d'Achille ou d'Agamemnon, celle d'Alexandre ou de César? Il est désormais temps que l'on conduise la jeune génération sur les lieux où leurs grands-pères ont acquis la couronne de laurier des guerres de libération, qui par leur importance historique ne peuvent se trouver que sur la page qui suit celle des grands héros des temps anciens ${ }^{(34)}$.

Passé une période durant laquelle le souvenir de la bataille de Leipzig fut soit oublié, soit repris en main au profit de la consolidation du pouvoir des princes allemands, l'image de la confrontation évolue en lien avec l'émergence de la légende napoléonienne. Durant les années 1840 et jusqu'aux années 1860, Leipzig est associé à une forme d'image des "temps héroïques " marqués par les guerres et par la figure de Napoléon. Le discours politique associé au récit des combats perd de son importance, désormais ce sont davantage les idéaux de libertés ou de changements qui sont mis en avant. À partir des années 1860, dans le contexte de l'attente de plus en plus forte d'une unification allemande, l'accroissement progressif de la puissance de la Prusse va contribuer à la création du caractère mythique de la bataille de Leipzig pour la nation allemande.

\section{La naissance d'un mythe national des années 1860 à 1871}

1863, l’année de la célébration du cinquantième anniversaire de la bataille de Leipzig, marque une étape importante pour la construction mémorielle de l'événement dans l'inconscient collectif allemand. Nourri par les espoirs d'unification nés en 1848 et par l'intérêt renouvelé pour la période napoléonienne, un véritable enthousiasme se développe en marge de l'organisation des festivités d'octobre 1863. Néanmoins, contrairement à 1814, les célébrations ne sont pas alors l'aboutissement d'un mouvement spontané mais le fruit de l'action menée par des partisans des idées nationales et libérales. Ces derniers voyant en l'organisation d'une fête l'occasion de " rassembler la bourgeoisie éclairée et patriote de toute l'Allemagne " à Leipzig afin de bâtir "l'unité et l'indépendance allemande » ${ }^{(35)}$. Dès 1862, un comité d'organisation centralisée se constitue. Il est placé sous la tutelle des municipalités de Leipzig et de Berlin qui lancent une invitation à toutes les villes d'Allemagne en septembre de la même année ${ }^{(36)}$. Ce choix ne fait cependant pas l'unanimité et plusieurs États de l'Allemagne méridionale préfèrent rester à l'écart de ces festivités trop influencées par la Prusse à leur goût. La Bavière, par exemple, ne répond pas à cette sollicitation: le 18 octobre 1863, le roi Maximilien II préfère inaugurer un monument dédié aux guerres de libération: la Befreiungshalle de Kelheim. Le chiffre 18, référence à la date du jour décisif de Leipzig ${ }^{(37)}$, est présent partout dans le programme iconographique développé

34 Theodor APPEL, Führer auf die Schlachfelders Leipzigs im Oktober 1813 und zu deren Marksteinen, Leipzig, Albert Hoffmann, 1863, 92 p.

35 Stefan-Ludwig Hoffmann, « Mythos und Geschichte, Leipziger Gedenkfeiern der Völkerschlacht im 19. und frühen 20. Jahrhundert », in: Étienne François, Hannes Siegrist, Jakok Vogel (dir.), Nation und Emotion, Deutschland und Frankreich im Vergleich 19. und 20. Jahrhundert, Göttingen, Vandenhoeck \& Ruprecht, 1995, p. 111-132.

36 Paul Daenne, «Die Gedenkfeier der Völkerschlacht vor fünfzig Jahren », Illustrirte Zeitung, 141/3669 (1913), p. 725.

37 Le chiffre 18 fait aussi référence au jour de la bataille de Waterloo: le 18 juin 1815. 
pour le bâtiment. Ce monument mesure 180 pieds de haut, à l'intérieur se trouvent 18 boucliers portant les noms des victoires remportées par la Coalition de Gross-Beeren à Waterloo ${ }^{(38)}$ et 18 représentations allégoriques de l'union des Allemands. Le roi de Bavière espérait ainsi renforcer le sentiment national de ses sujets et démontrer que les Bavarois se trouvaient en première ligne au moment où les Allemands ont combattu Napoléon. L'impact de cette inauguration fut mince; dans sa correspondance avec le ministère français des Affaires étrangères, l'ambassadeur de France à Munich laisse entendre que les célébrations sont restées limitées:

"Le temple élevé par le roi Louis [sic] en l'honneur des généraux qui ont combattu contre la France en 1813 et 1814 a été inauguré avec un grand éclat le 18 octobre. On s'étonne que sa majesté ait choisi pour cette cérémonie un pareil anniversaire, car, si je ne me trompe, ce n'est qu'après la bataille de Leipsick que les troupes bavaroises se sont rangées parmi nos adversaires. Du reste, la fête commémorative qui vient d'être célébrée dans toute l'Allemagne n'a eu qu'un demi-succès à Munich parce que son caractère n'était pas suffisamment déterminé et qu'on y a vu surtout une menace du parti démocratique ${ }^{(39)}$.

L'ambassadeur en Bavière perçoit ainsi une attitude ambiguë au sujet de la bataille de Leipzig, entre volonté de récupération et prudence politique. De surcroît, les ambitions du roi de Bavière ne correspondent plus aux attentes d'alors des festivités prévues dans la ville saxonne en octobre 1863. Maximilien II inscrit sa démarche dans celle des monarques qui, au début $\mathrm{du} \mathrm{XIX}^{\mathrm{e}}$ siècle, ont voulu orienter le souvenir des guerres de libération à leur profit. Or, au contraire, les festivités de Leipzig cherchent non pas à exalter tel ou tel prince mais bien à renforcer le sentiment national et libéral d'une partie des Allemands. Dans ses lettres, l'ambassadeur de France auprès du roi de Saxe donne des exemples de ces divergences et des craintes suscitées par les événements en préparation:

"Concernant la célébration du $50^{\mathrm{e}}$ anniversaire de la bataille de Leipsick. La démonstration aura lieu, mais les auteurs et ceux qui s'y étaient associés doivent aujourd'hui regretter de s'être trop avancés pour pouvoir reculer. Le nombre d'abstention des villes allemandes augmente chaque jour et il se mêle à ces abstentions des incidents de nature à tourner en ridicule tout ce qui se fera. Par exemple, il est telles villes qui en renvoyant l'invitation qui leur est adressée, en réclament les frais de poste. Le gouvernement saxon vient de défendre à tout fonctionnaire, sous peine de destitution de participer à quoi que ce soit de la célébration. S'il eût pu faire davantage, il n'aurait pas manqué à ses devoirs. Mais à tort ou à raison il a craint en s'opposant à toute démonstration, d'aller au-devant des désirs des meneurs qui se plaignent de rencontrer trop de libertés et pas assez de martyrs. C'est, il est vrai, en usant de cette excuse qu'on a laissé faire de grands pas aux partis exaltés et qu'on se trouve aujourd'hui vis-à-vis d'eux dans de grands embarras dont on ne sortira qu'avec beaucoup d'énergie et de prudence. Le gouvernement saxon dans sa défense aux fonctionnaires de prendre part, sous aucun prétexte, aux fêtes de Leipsick rappelle sa déclaration d'il y a quelques mois, que tout en refusant de prendre l'initiative de la célébration de l'anniversaire de la bataille, il s'y associerait, si cette démonstration prenait un caractère national. Or, c'est ce qu'elle n'a pas en présence de l'abstention de tant de villes allemandes. Tous les Saxons, un peu sensés, qui sentent tout ce qu'il y a d'humiliant pour leur pays

38 «Die Befreiungshalle bei Kelheim », Illustrirte Zeitung, 41/1060 (1863), p. 295-298, ici p. 295.

39 Archives du ministère des Affaires Étrangères, correspondance politique jusqu'en 1896, Bavière, vol. 238 (1863), Reculot, 30 octobre 1863. 
et surtout pour leur armée dans les journées des 17, 18 et 19 octobre 1813, sont opposés à toute démonstration. Les vieux vétérans se livrent entre eux, à ce sujet, à des discussions qui remplissent certaines feuilles [...]. Le gouvernement saxon a pris des mesures pour réprimer tout désordre; et si je ne me trompe, les troupes destinées au Holstein pourraient bien, au besoin, aider à rétablir l'ordre, s'il venait à être troublé dans la ville de Leipsick où le parti modéré commence à s'organiser de manière à pouvoir venir bientôt en aide au gouvernement ${ }^{(40)}$.

La prudence est de mise, mais les célébrations du cinquantième anniversaire de Leipzig débutent bien le 16 octobre $1863^{(41)}$. La fête rencontre un immense succès, des milliers de personnes ont convergé vers Leipzig. L'Illustrirte Zeitung évoque une foule rassemblant plus de 100000 participants réunis dans une immense liesse autour du drapeau rouge-noir-or et des vétérans des guerres napoléoniennes ${ }^{(42)}$. Durant ces journées, la pièce de Kleist, Die Hermannschlacht, est à l'honneur. Cette œuvre, mettant en scène le mythe de la bataille de Teutobourg remportée par Arminius, est reprise par les organisateurs de la fête du cinquantenaire. L'assimilation voulue par Kleist entre les antagonistes Romains et Germains en parallèle aux Français et aux Allemands concorde avec les ambitions affichées pour cette fête. La pièce écrite en 1808 rencontre un grand succès. Près de soixante ans plus tard, les nationalistes allemands sont sensibles à l'exaltation du sentiment d'appartenance à la nation allemande notamment dans des vers comme: "Ô puissions-nous bientôt fêter et célébrer le bonheur d'une plus glorieuse victoire! [...] puissions-nous, ô Hermann, bander l'arc d'airain de la guerre, et de nos forces unies lancer le trait de la bataille, et fracasser la nuque de l’armée romaine qui a pris racine dans la campagne allemande! » ${ }^{(43)}$ Les cérémonies mettent également à l'honneur le rôle joué par les troupes prussiennes, notamment lors de la pose de la première pierre d'un « monument national » ${ }^{(4)}$ destiné à commémorer la bataille de Leipzig (en l'absence de volonté politique et, de manière plus prosaïque, du manque de financement, le projet demeurera sans suites dans l'immédiat). Le succès remporté par ces deux journées suscite de grands espoirs. Le journal cité précédemment espère que « la fête allait faire croître un mouvement dont l'aboutissement serait l'accomplissement d'un rêve inaccessible: une entière, une unique Allemagne! » ${ }^{(45)}$ Dans le contexte des années 1860 et après les événements de 1848 , ces fêtes sont une véritable tribune pour les partisans de l'unité politique allemande. De fait, la Prusse, voyant tout le bénéfice qu'elle pourrait en retirer, joue un rôle essentiel dans l'organisation des festivités. Cette place privilégiée offerte à la Prusse se traduit d'ailleurs par l'absence d'autres membres de la Coalition de 1813-1814 lors des célébrations.

40 Arch. du ministère des Aff. Étrangères, correspondance politique jusqu'en 1896, Saxe électorale et royale, vol. 135 (sept-déc. 1863), M. Forth-Rouen, 13 octobre 1863.

41 Jérôme Schweitzer, "La commémoration du cinquantième anniversaire de la bataille de Leipzig ", Revue de l'Institut Napoléon, 207 (2013), p. 93-102.

42 « Die funfzigjährige Gedenkfeier der Völkerschlacht bei Leipzig », Illustrirte Zeitung, 41/1060 (1863), p. 299.

43 Heinrich von Kleist, La bataille d'Arminius, un drame (Jean-Louis Besson, Jean Jourdheuil, trad.), Paris, Éd. théâtrales, 1995, p. 13.

44 S. Poser, Denkmale zur Völkerschlacht (note 18), p. 30.

45 «Die funfzigjährige Gedenkfeier der Völkerschlacht bei Leipzig » (note 42), p. 322. 
L'Autriche, la Russie ou la Suède sont à peine mentionnées lors des cérémonies, entamant ainsi un véritable processus d'accaparement du prestige lié à la bataille de Leipzig au seul profit de la Prusse. Dès lors, cette dernière est élevée au rang de héraut de l'unification de l'Allemagne et les récits de la bataille de Leipzig donnent la part belle au rôle estimé décisif qu’auraient joué les troupes menées par Blücher.

Mais cette place revendiquée par la Prusse n'obtint pas l'aval de l'ensemble des monarques allemands: les princes bavarois, badois ou wurtembergeois jettent un regard indifférent voire hostile sur les fêtes de Leipzig de 1863. À l'instar du grand « monument national » resté au stade de l'ébauche, les retombées politiques immédiates de cette commémoration sont limitées à l'échelle de l'Allemagne. Finalement après les guerres d'unification, c'est l'achèvement de l'unité allemande sous l'égide prussienne et le besoin de créer des lieux où se cristalliserait la mémoire nationale qui permit la construction du grand monument de Leipzig, inauguré en 1913. Les festivités du centenaire marquent ainsi l'aboutissement de "l'élan patriotique » réputé être né lors de la «bataille des Nations » de 1813.

\section{Résumé}

En octobre 1813, la nouvelle de la défaite de Napoléon dans les plaines qui entourent Leipzig déchaîne les passions en Allemagne. Profitant de l'absence de pouvoir fort, les partisans de la création d'un État regroupant l'ensemble des Allemands s'emparent du récit des combats pour organiser son souvenir. La Restauration mettra un terme à ce premier mouvement spontané, il faut ensuite attendre les retombées du Printemps des peuples de 1848, pour voir se réactiver le souvenir de Leipzig. La Bavière et la Prusse se disputent d'ailleurs un temps la mémoire de l'événement avant que, lors des fêtes de 1863 commémorant le cinquantième anniversaire de la bataille, la Prusse ne parvienne à faire tourner les événements à son avantage. Finalement la proclamation de l'Empire allemand en 1871 puis surtout les festivités du centenaire de la bataille de Leipzig seront le point final de cet accaparement du souvenir de la bataille par la Prusse.

\section{Zusammenfassung}

Die Nachricht von der Niederlage Napoleons in den Ebenen um Leipzig im Oktober 1813 bewirkte in ganz Deutschland scharfe Emotionen. Die Anhänger der deutschen Einheit nutzten die relative Redefreiheit aus, um das Gedächtnis der Schlacht zu organisieren. Nach 1818 setzte die konservative Reaktion dieser ersten spontanen Bewegung ein Ende, erst im Vormärz wurde die Erinnerung an Leipzig reaktiviert. Bayern und Preußen konkurrierten um das Andenken an das Ereignis, bis anlässlich der Feierlichkeiten von 1863 zum fünfzigsten Jubiläum der Schlacht Preußen die Veranstaltungen zu seinem Vorteil nutzen konnte. Schließlich bilden die Proklamation des Deutschen Reichs im Jahr 1871 und die Feierlichkeiten zum hundertsten Jahrestag der Völkerschlacht bei Leipzig der Schlusspunkt dieser Monopolisierung der Erinnerung an die Schlacht durch Preußen. 